\title{
Las redes sociales y herramientas de publicación de anuncios digitales para mejorar la promoción de servicios arquitectónicos en Loja
}

\section{Social networks and ads promoting tools to improve promotion of architectural services in Loja city}

\author{
Fredy Mauricio Cueva-Bravo \\ Universidad Internacional del Ecuador, Ecuador
}

Autor para correspondencia: frcuevabr@uide.edu.ec

Fecha de recepción: 20 de julio de 2018 - Fecha de aceptación: 30 de agosto de 2018

Resumen: La presente investigación es un trabajo realizado en el sector servicios arquitectónicos de la ciudad de Loja-Ecuador, cuyo propósito es mostrar el potencial de las redes sociales y herramientas de publicación de anuncios como impulsadoras de la venta de servicios en el sector arquitectónico. Las escasas y subutilizadas redes sociales, así como las herramientas de publicación de anuncios usadas por el arquitecto dueño de la empresa Arista Construcciones, fueron creadas de tipo familiar, de comunicación con amigos y familiares, más no con propósitos de promocionar los servicios que tienen, evidenciándose en un inicio 228 seguidores, poca interacción de usuarios en su página de Facebook, y reducido número de ventas de sus servicios. La metodología empleada fue la entrevista, observación directa y el método deductivo. Los principales resultados muestran un aumento del número de Likes a 2683 mejorando el posicionamiento digital de la marca en el mercado local, también incrementó el número de interacciones/acciones de los usuarios con las publicaciones y por ende el número de ventas y solicitudes de los servicios provistos por la empresa. Se concluye que el uso de las redes sociales y herramientas de publicación de anuncios digitales impactan en el posicionamiento digital y la mejora de la promoción de servicios en el sector arquitectónico en la ciudad de Loja-Ecuador.

Palabras clave: redes sociales; promoción; facebook; google negocios; publicidad

Abstract: The present research is a work carried out in the architectural services sector of the city of Loja-Ecuador, which purpose is to show the potential of social networks and ads publication tools as promoters of the sale of services in the architectural sector. The scarce and underutilized social networks, as well as the ads publishing tools used by the architect-owner of the company Arista Constructions were created as a family type page, for communication with friends and family, but not for the purpose of promoting the services they have, having at beginning 228 followers, little interaction of users in their Facebook Fan Page, and reduced number of sales of their services. The methodology used was the interview, direct observation and the deductive method. The main results show an increase in the number of Likes to 2683, improving the digital brand positioning in the local market, the number of interactions / actions of the users with the contents and therefore the number of sales and services provided by the company and requested by users increased as well. It is concluded that the use of social networks and digital ads publishing tools impact on the brand digital positioning and the improvement of the promotion of services in the architectural sector in the city of LojaEcuador.

Key Words: Social Network, promotion, Facebook, google business, marketing 


\section{Introducción}

El proyecto de trabajo se realizó en el sector de servicios arquitectónicos y de construcción en la ciudad de Loja-Ecuador, se originó como una iniciativa del docente a cargo de la clase de E-business y Marketing por Internet orientado a trabajar con redes sociales para mejorar el posicionamiento de la empresa, la promoción de este tipo de servicios y el incremento de ventas o clientes que hacen uso de los mismos.

La investigación constituye un trabajo académico y de cooperación de clases entre los estudiantes, el Gerente Propietario de la empresa y el docente a cargo de la asignatura de Ebusiness y Marketing por Internet de la Universidad Internacional del Ecuador extensión LojaEcuador, orientado a trabajar con redes sociales como herramientas que mejoran la promoción y ventas de este tipo de servicios.

El estado del arte de esta investigación se enfoca en que las redes sociales, como herramientas con visión de comunidad y creación de contenido atractivo que permiten mejorar la interacción con los usuarios o prospectos de clientes, se llega a mantener el interés de los usuarios, incrementar su número y finalmente incrementar las ventas de los servicios o productos. (Weber, 2010, pág. X)

Las redes sociales brindan la posibilidad de acceder a mercados distintos alrededor del mundo, miles de millones de personas que están dispuestas a comprar o vender productos o servicios y tomar ventaja de estos mercados.

La redes sociales a diferencia del marketing tradicional, mejora la interacción de los empresas y sus clientes virtuales con las páginas empresariales, permitiendo de mejor forma a los usuarios acceder a productos o servicios que están cerca de su área geográfica, usando segmentación de mercados, mientras que por otro lado a los empresarios les permite ubicar a sus clientes potenciales, cercanos al lugar de la compra o de localización de la empresa, volviendo más eficiente el proceso de adquisición de nuevos usuarios o clientes y facilitando las ventas; lo anterior sin tomar en consideración que les permite acceder a esos clientes que antes estaban aislados por el mismo hecho geográfico y que hoy gracias al internet y la estructura de las redes sociales y herramientas de publicación de anuncios muchas barreras físicas se desaparecen. (Weber, 2010)

Por otro lado los costos de operación, tanto para el cliente como para el empresario se reducen, lo que anteriormente de forma tradicional, encontrar un buen servicio era cosa de las redes reales de amigos que se tenían a disposición, actualmente, debido a la interacción que existe entre las redes sociales más conocidas como Facebook, google maps, google negocios, Instagram, twitter, YouTube, LinkedIn, snapchap, se puede observar la interacción que los usuarios tienen con un negocio, la calificación y los comentarios que le dan al mismo, haciendo que claramente el cliente virtual que navega en Internet entienda claramente el valor del servicio o producto que va a obtener, acorde a la calificación otorgada por otros clientes virtuales que lo han usado previamente.

Las redes sociales más utilizadas a nivel mundial son el Facebook, Instagram, LinkedIn, twitter, Bing, Google, y herramientas de digitales de publicación hay muchas en la internet, 
algunas gratuitas y otras pagadas. De manera sencilla se puede entender que la popularidad de la red social será la clave para que el impacto en los resultados de la publicación sea mayor o menor. La red social Facebook viene siendo pionera en esta área desde el 2011 (Barker, Barker, Bormann, \& Neher, 2015) hasta la actualidad, datos que se contrastan con la información encontrada en la web, respecto del ranking de las redes sociales que se muestra a continuación.

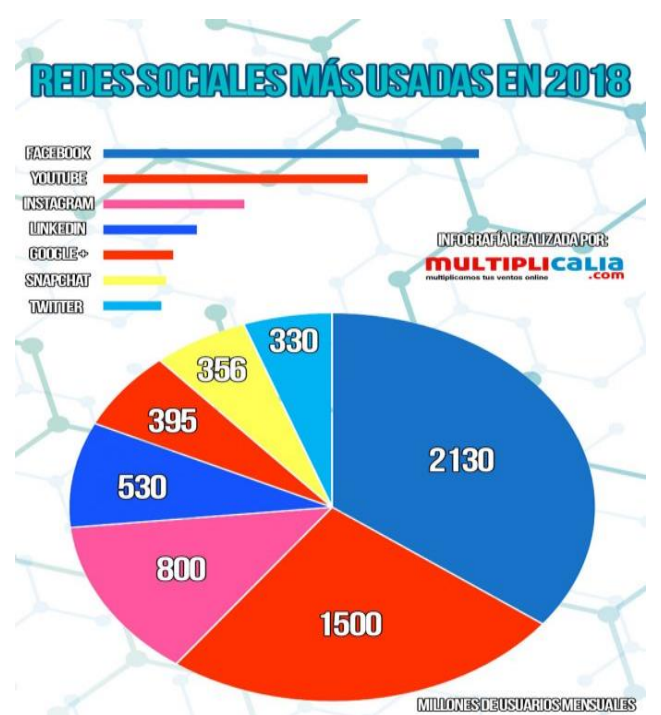

Fuente: Multiplicalia. (2018). Multiplicalia.

Obtenido de https://www.multiplicalia.com/redes-sociales-mas-usadas-2017/

\section{Marketing de redes sociales}

Barker, Barker, Bormann, \& Neher, (2015) afirma: que realizar marketing en medios sociales como medio de mejorar la promoción de contenido empresarial "Es un término que describe el uso de redes sociales, comunidades en línea, blogs, wikis y cualquier otro medio de colaboración en línea en actividades de marketing, ventas, relaciones públicas y servicios al cliente."

El propósito de realizar marketing de medios sociales es generar "ruido" en los medios de tal forma que la imagen de la empresa, producto o servicio sea afectada positivamente por las publicaciones realizadas, haciendo de está, una principal atracción para el consumidor final.

Según Gómez Otero (2014), define a las redes sociales como "un lugar de encuentro para varios miles o incluso millones de personas de todo el mundo, que comparten unos intereses y/o características sociodemográficas, y que participan en un proceso de comunicación y de difusión de todo tipo de contenidos y noticias."

Dentro de muchos de los casos exitosos al momento de realizar marketing en medios sociales y con herramientas de publicación de anuncios, están expresados según Kutchera (2014), como modelos a seguir para el desarrollo de plan de marketing de medios sociales.

Se manifiesta que el uso de las redes sociales y las herramientas de publicación por internet es incrementar la interacción con los usuarios o prospectos de clientes, interés de los 
usuarios respecto de los servicios o productos, número clientes potenciales y reales y las ventas de los servicios o productos.

\section{Medición y análisis de resultados en Marketing Digital}

La medición de resultados es de suma importancia al momento de establecer hitos para la verificación del avance de un plan de promoción de productos o servicios o de un plan de marketing. Según lo establece Crespo (2016) "si no se pueden cuantificar los beneficios obtenidos respecto de los resultados esperados” no sirve de nada. (Martínez, 2015, pág. 43)

El internet como medio digital, permite que a través de sus herramientas se pueda hacer un estudios minucioso de lo que se va generando mientras el empresario/emprendedor desarrolla sus estrategias de marketing digital. Dentro de las mediciones podemos establecer, la medición del número de visitas realizadas a la página fan page de la empresa, la interacción que los usuarios tienen con el contenido que estamos generando, el impacto de los contenidos, etc. (Crespo, 2016) \& (Rodríguez del Pino \& Miranda Villalón, 2012) (Domínguez Doncel, 2010, pág. 47)

\section{Las herramientas de publicación de anuncios clasificados digitales}

Las herramientas de publicación de anuncios digitales o conocidos como anuncios clasificados, son plataformas digitales que permiten a las compañías, empresarios, o clientes poder acceder a espacios de publicación digitales de manera gratuita y rápida, dónde ellos pueden promocionar sus productos o servicios de forma segregada o segmentada, rápida y sencilla, aumentando sus posibilidades de alcanzar nuevos clientes y todo esto a través del uso de nuevas tecnologías de comunicación como dispositivos móviles sus computadores personales. (OLX, 2018)

Una plataforma de publicación de anuncios clasificados es más importante que otra cuando llega a más países alrededor del mundo, y la cantidad de visitas a la misma o la popularidad que tiene entre los usuarios de internet es alta.

Ejemplos de este tipo de plataformas están: OLX, MercadoLibre, Doplim, milanuncios.com, wallapop.com, vibbo.com, locanto.es, plusvalía, trovit, blidoo, multitrabajos, patiotuerca, patiosdeautos.com, mitula, eavisos, opcionempleo, eBay, computrabajo, etc., variando su popularidad e impacto en la promoción, acorde a la región donde se encuentra el usuario, empresario o compañía que desea realizar la publicación y de la misma manera varía su impacto. Los siguientes son una lista de los top 10 plataformas de publicación de anuncios clasificados en Ecuador y su comparativa con España.

\begin{tabular}{cll}
\hline Ranking & Ecuador & España \\
\hline $\mathbf{1}$ & Anuto.ec & Anuto.es \\
$\mathbf{2}$ & Olx.com.ec & Milanuncios.com \\
$\mathbf{3}$ & Locanto.com.ec & Wallapop.com \\
$\mathbf{4}$ & Mercadolibre.com.ec & Vibbo.com \\
\hline
\end{tabular}




\begin{tabular}{crl}
\hline 5 & Doplim.ec & Locanto.es \\
6 & Blidoo.com.ec & Vivalocal.es \\
7 & Evisos.ec & Tablondeanuncios.com \\
8 & Anuxi.ec & Merkatia.com \\
9 & Anunico.ec & Anuncios.es \\
10 & Segundamano.ec & Letgo.com \\
\hline
\end{tabular}

Fuente: (ANUTO.NET, 2018), (ANUTO.NET Ecuador, 2018)

\section{E-Commerce}

El marketing por internet se puede hacer a través de diferentes redes sociales, siendo que la parte inicial para poder establecer una buena comunicación entre clientes y empresas o emprendimientos, es el desarrollo del e-Commerce, definiéndolo a este según Cisneros (2016), como la "actividad económica basada en el ofrecimiento de productos o servicios para la compra o la venta, a través de medios digitales como internet".

\section{El Marketing y Publicidad en Internet}

El marketing y publicidad en internet, es el conjunto de acciones orientadas a establecer objetivos estratégicos, operativos y sus principales acciones a desarrollar, para hacer de ellas una oportunidad grande para las empresas y micro empresarios que necesitan de este tipo de publicidad. Desde el principio del marketing se busca entregar un producto o servicio al consumidor con mayor valor, coste adecuado, de forma conveniente y a través de una comunicación adecuada, y desde el punto de vista de la publicidad se pretende hacer llegar al consumidor un contenido de calidad, atractivo, que impacte y que genere en los clientes un sentido de comunidad y de conectividad que permita promocionar productos o servicios de mejor forma e incrementar las ventas. (Martí Parreño, 2011)

\section{Internet como Medio Publicitario}

El hecho de establecer al internet como un medio publicitario para las pequeñas y grandes empresas y micro-emprendimientos, es que permite entregar a los clientes reales y potenciales una publicidad con valor añadido, generar interacción entre los que pertenecen a las redes sociales o a aquellos que usan las herramientas de creación de anuncios publicitarios para la promoción de productos y servicios. A más de esto nos permite personalizar los mensajes para una audiencia o el mercado objetivo adecuado, desarrollar desde el push al pull y sobre todo tener mayor presencia dentro del mercado segmentado. (Martí Parreño, 2011) (Serrano, 2005, pág. 15)

\section{Redes sociales para la generación de clientes potenciales}

Diferentes empresas alrededor del mundo están usando redes sociales para realizar sus negocios, promocionar productos o servicios, ejemplos de aquello es el caso de LinkedIn, compañía que usó las redes sociales para posicionar su marca, productos y generar clientes potenciales. (Barker, Barker, Bormann, \& Neher, 2015, pág. 190) 
Según Gómez \& Otero (2013), "las redes sociales son un fenómeno mundial, una topología relacional entre personas, puesto que se establecen canales de comunicación estables entre ellas con el objeto de difundir novedades, noticias o simplemente estados de ánimo" pero que en si a la final del día, permiten a los empresarios establecer comunicación directa con los clientes e incrementar sus ventas.

El marketing en redes sociales es usado mayoritariamente para realizar un buzz al mercado digital objetivo, que permita llamar la atención de los espectadores, en este caso, de aquellos que están buscando por el producto o servicio que estamos ofertando. (Ugarriza, 2013)

Una de las grandes razones por las cuales actualmente se usa los medios sociales en la mayoría de aplicaciones de negocios, es debido al hecho de poder llegar a un vasto número de consumidores en el accionar de un simple clic, consumidores que anteriormente debíamos incrementar un esfuerzo monumental para poder alcanzarlos. (Barker, Barker, Bormann, \& Neher, 2015, pág. 3) (Gómez Vieites \& Otero Barros, 2013, pág. 275)

\section{Finalidad}

Objetivo General: Determinar el impacto de las redes sociales y las herramientas de publicación de anuncios en la promoción de los servicios arquitectónicos en la ciudad de Loja.

Hipótesis: La inclusión de contenido de calidad en redes sociales y el incremento en la cantidad de publicaciones o anuncios digitales clasificados generados por la empresa, incrementará mayor la interacción y presencia de los usuarios empresa y por ende mejorará la promoción de sus servicios.

Variables Dependientes: Interacción de las redes sociales, incremento en número de fans en la fan page, Incremento de búsquedas en Google Search, incremento de Ventas.

Variables Independientes: Contenido de calidad, incremento en número de publicaciones, generación de interacciones con usuarios.

\section{Metodología}

La metodología utilizada en esta investigación es de tipo cuantitativa-cualitativa, empleando la entrevista, observación directa y el método deductivo. Cuantitativa al momento de usar las herramientas de medición de métricas digitales de Facebook y de Google Negocios de la empresa en mención. La información fue obtenida del trabajo de clase desarrollado en la asignatura de E-business y Marketing por Internet, la misma que fue esquematizada para propósitos de documentar cuantitativamente y cualitativamente este artículo, mostrando resultados comparativos de la línea base y el momento actual en el que se encuentra la compañía. 


\section{Resultados}

Los resultados obtenidos son de tipo comparativos, en la cual se usó la observación directa, y el método deductivo para obtenerlos, mostrando como más relevantes los siguientes:

- El uso de la red social Google mi negocios enlazado a Facebook permitió que las usuarios conozcan de la calificación que los clientes han dado al servicio de la empresa.

- La incidencia de usar la red social de Google mi negocios permitió acceder a nuevos canales de promoción, de manera directa los clientes potenciales pueden buscar la empresa de forma directa.

- Solicitar de manera orgánica la realización de clics, desde el perfil del Gerente de la empresa, se obtiene porcentajes más altos de alcance de publicaciones y menores en rechazo.

- Las invitaciones orgánicas desde otros perfiles de Facebook a dar like a la fan page empresarial, no dio resultados favorables.

- El incremento de interacciones con mensajes personalizados a través de Facebook Messenger tuvo una alta acogida.

- La actualización de la foto de perfil de la empresa como generación de contenido y mejora de la compañía tuvo un alcance relativamente alto.

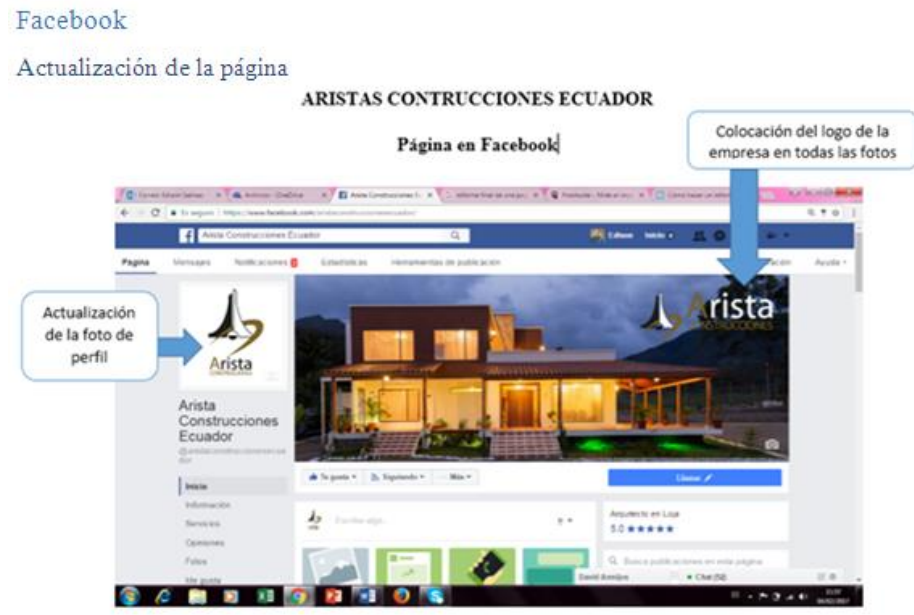

Fuente: Obtenida de la herramienta de Facebook Fan Page, (2017).

- Resultados de fotos: En la foto de perfil se ubicó el logo de la empresa y fue subida el 4 de diciembre del 2016, la cual tuvo un total de 15 likes, 19 personas alcanzadas y 1 compartida.

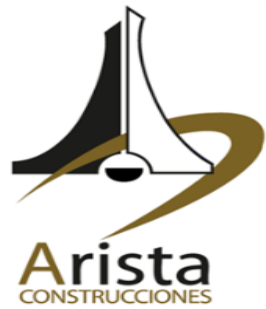

Fuente: Obtenida de la herramienta de Facebook Fan Page, (2017). 
- La foto de portada seleccionada como generadora de contenido fue una casa diseñada y construida por el arquitecto, Gerente General, la cual obtuvo al 3 de diciembre del 2016 un total de 20 likes, 743 personas alcanzadas y 1 una vez compartida.

- Las publicaciones realizadas por el Gerente General antes del 2015, previo al proceso de mejora masivo, tenían cero interacciones con los usuarios:

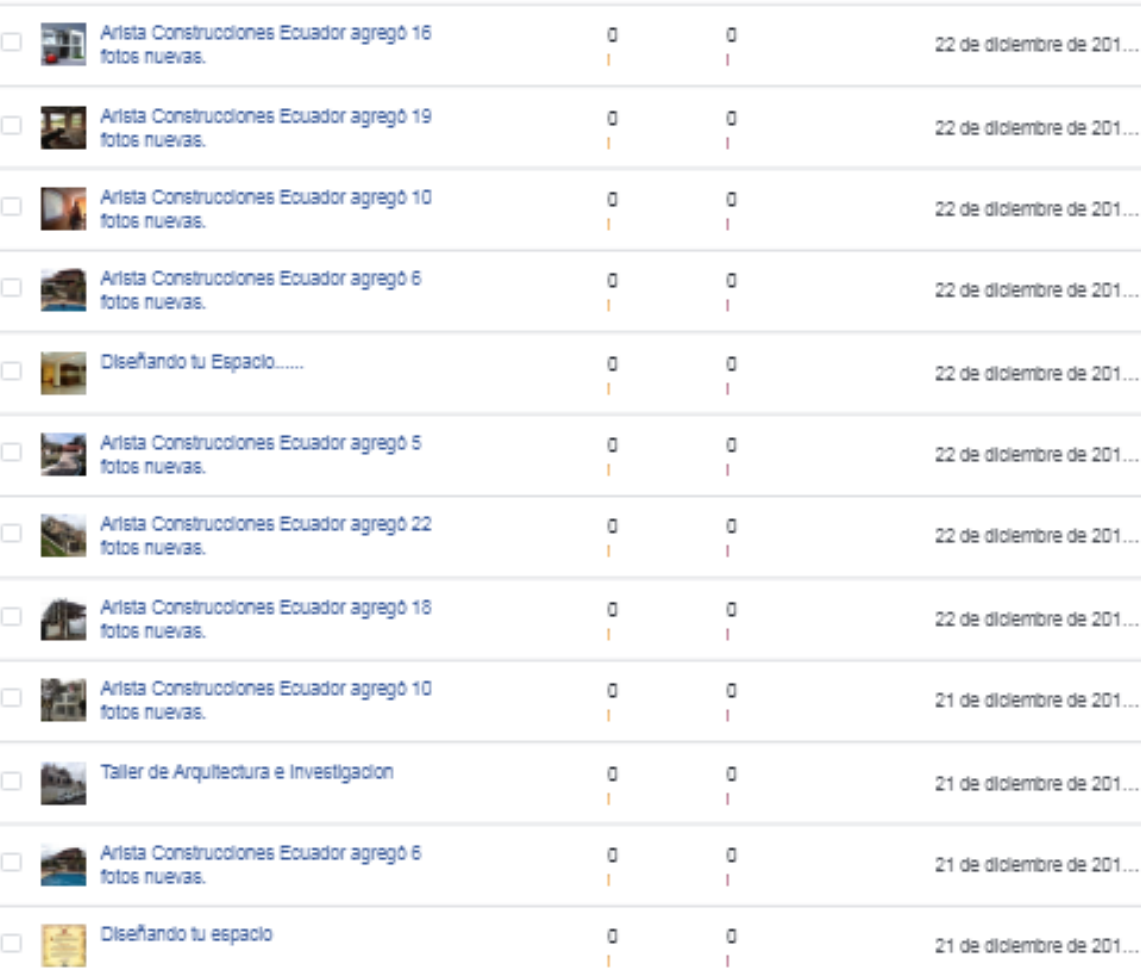

Fuente: Métricas obtenidas de la herramienta de Facebook Fan Page, (2017)

- Las publicaciones realizadas después de agosto de 2016, posterior al uso de las redes sociales y las herramientas de publicación de anuncios tienen resultados elevados en alcance e interacción (clics y o acciones). 


\begin{tabular}{|c|c|c|c|c|}
\hline$\square$ & Publicaclones & Alcance & Clics/acoliones & Publicadas \\
\hline$\square$ & $\begin{array}{l}\text { Arlsta Construcdiones Ecuador actualizo } \\
\text { su foto de portada. }\end{array}$ & 327 & 95 & $\begin{array}{l}7 \text { de enero de } 2017 \text { a la... } \\
\text { David Avmljos }\end{array}$ \\
\hline & $\begin{array}{l}\text { Arlsta Construcciones te desea una Felz } \\
\text { Navidad y un Prospero Aflo Nuevo }==\end{array}$ & 194 & 16 & $\begin{array}{l}22 \text { de diclembre de } 201 . . . \\
\text { Marta Claudla Castro } \\
\text { Andrade }\end{array}$ \\
\hline$\square$ & $\begin{array}{l}\text { Aclsta Construcciones te desea una Felz } \\
\text { Navidad y un Prospero Aflo Nuevo! }\end{array}$ & 794 & 58 & $\begin{array}{l}22 \text { de dlolembre de } 201 . . . \\
\text { Marta Claudla Castro } \\
\text { Andrade }\end{array}$ \\
\hline & $\begin{array}{l}\text { Arlsta Construcciones te entrega la llave } \\
\text { para hacer tus suehos realidad. }\end{array}$ & $1.1 \mathrm{~K}$ & 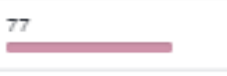 & $\begin{array}{l}13 \text { de didembre de } 201 . . \\
\text { Marta Claudla Castro } \\
\text { Andrade }\end{array}$ \\
\hline & $\begin{array}{l}\text { Arista Construcciones.. Especial } \\
\text { atencion en los detales }\end{array}$ & 646 & 68 & $\begin{array}{l}6 \text { de diclembre de } 2016 \text {... } \\
\text { Fernando E Fher } \\
\text { Jaramillo Palacios }\end{array}$ \\
\hline & $\begin{array}{l}\text { =Avista \#Construcciones fgir } \\
\text { \#AvistaConstrucdionesEc... }\end{array}$ & 581 & 43 & $\begin{array}{l}4 \text { de diclembre de } 2016 \ldots \text {... } \\
\text { David Avmijos }\end{array}$ \\
\hline & $\begin{array}{l}\text { Arlsta Construcdiones Ecuador actualzo } \\
\text { su foto del perni. }\end{array}$ & 20 & 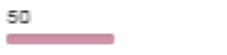 & $\begin{array}{l}4 \text { de diclembre de } 2016 \ldots \\
\text { David Avmljos }\end{array}$ \\
\hline & $\begin{array}{l}\text { Arlsta Construcdiones Ecuador actualizo } \\
\text { su foto de portada. }\end{array}$ & 743 & 96 & $\begin{array}{l}3 \text { de didembre de } 2016 \ldots \\
\text { David Avmljos }\end{array}$ \\
\hline & $\begin{array}{l}\text { Arlsta Construcdiones Ecuador agrego } \\
\text { una foto nueva - en Lola. }\end{array}$ & 373 & 28 & 3 de diclembre de $2016 \ldots$ \\
\hline$\square$ & La Arquntectura _.....ml pasion & 127 & 10 & $\begin{array}{l}30 \text { de nowembre de } 201 . . . \\
\text { Fernando E Fher } \\
\text { Jaramillo Palacios }\end{array}$ \\
\hline & $\begin{array}{l}\text { 19. Cludad y Academla un compromiso de } \\
\text { particlpacion.... }\end{array}$ & 306 & 84 & 17 de noviembre de $201 \ldots$ \\
\hline & EL DISENO CONSTRUYY SUENOS & 350 & 84 & 29 de agosto de $2016 \mathrm{a}$. \\
\hline$\square$ & EFECTOS DE LUZ Y SOMBRA & a & a & 2 de junio de 2016 a las... \\
\hline
\end{tabular}

Fuente: Métricas obtenidas de la herramienta de Facebook Fan Page, (2017)

Fuente: Proyecto desarrollado dentro de la clase.

- Las visitas generadas a la fan page de Facebook incrementaron cuando se generó el contenido de calidad y el número de publicaciones creadas aumentó, de 0 a 651 su pico máximo. El proceso se realizó desde agosto 2016 hasta febrero del 2017.

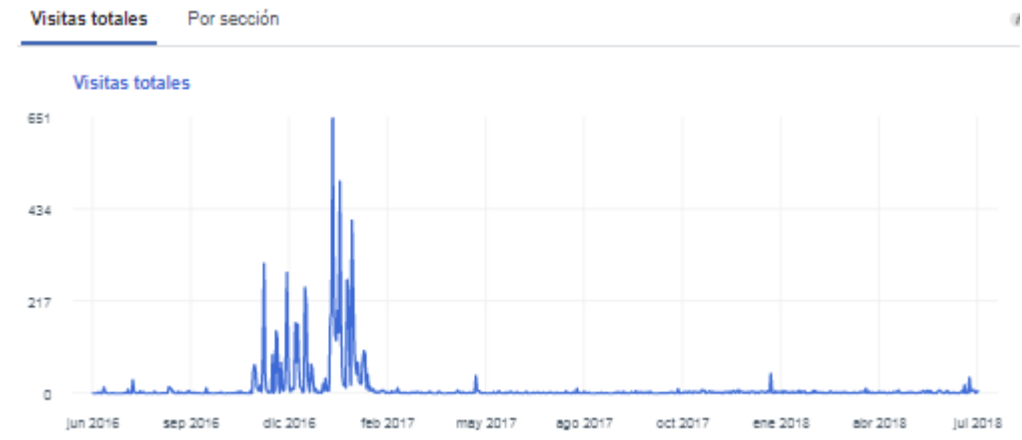

Fuente: Métricas obtenidas de la herramienta de Facebook Fan Page (2017)

- El total de visitantes en la fan page en el periodo antes mencionado, incrementó de 0 a 513 el pico máximo. 


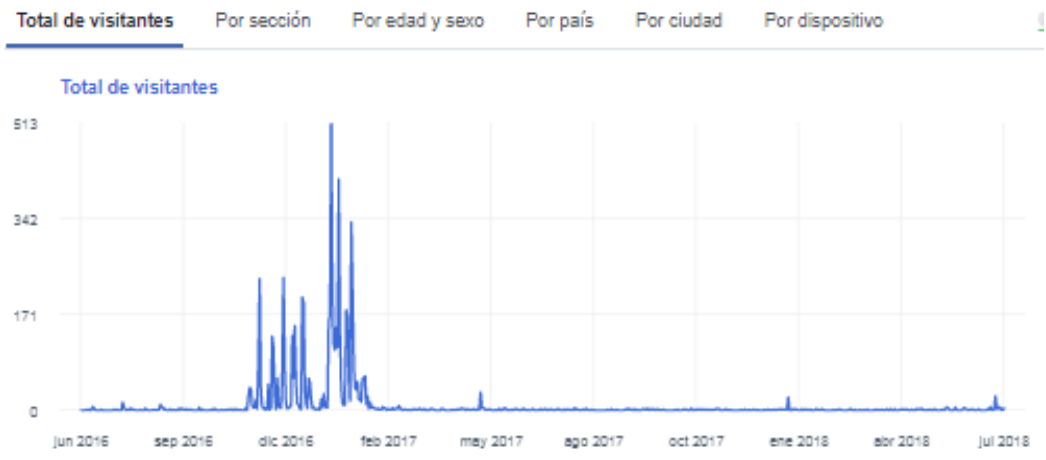

Fuente: Métricas obtenidas de la herramienta de Facebook Fan Page (2017)

- La conectividad entre redes sociales, Google mi negocio y Facebook fan page hizo posible que a la empresa la encuentren más clientes a través de la red social, teniendo búsquedas a un pico máximo de 94 en los mismos periodos anteriores.

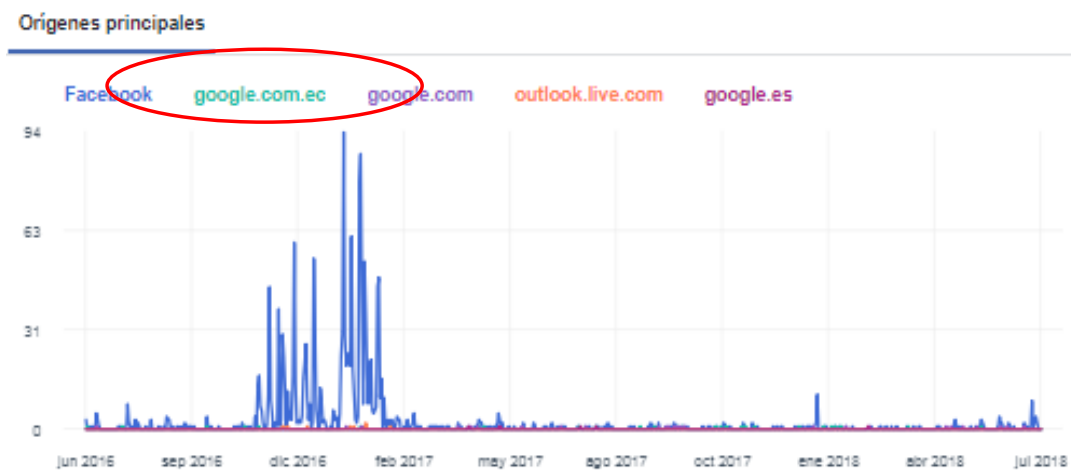

Fuente: Métricas obtenidas de la herramienta de Facebook Fan Page (2017)

- Los contenidos que tienen mayor interacción son aquellos relacionados a la actividad a la que se dedica el arquitecto. 


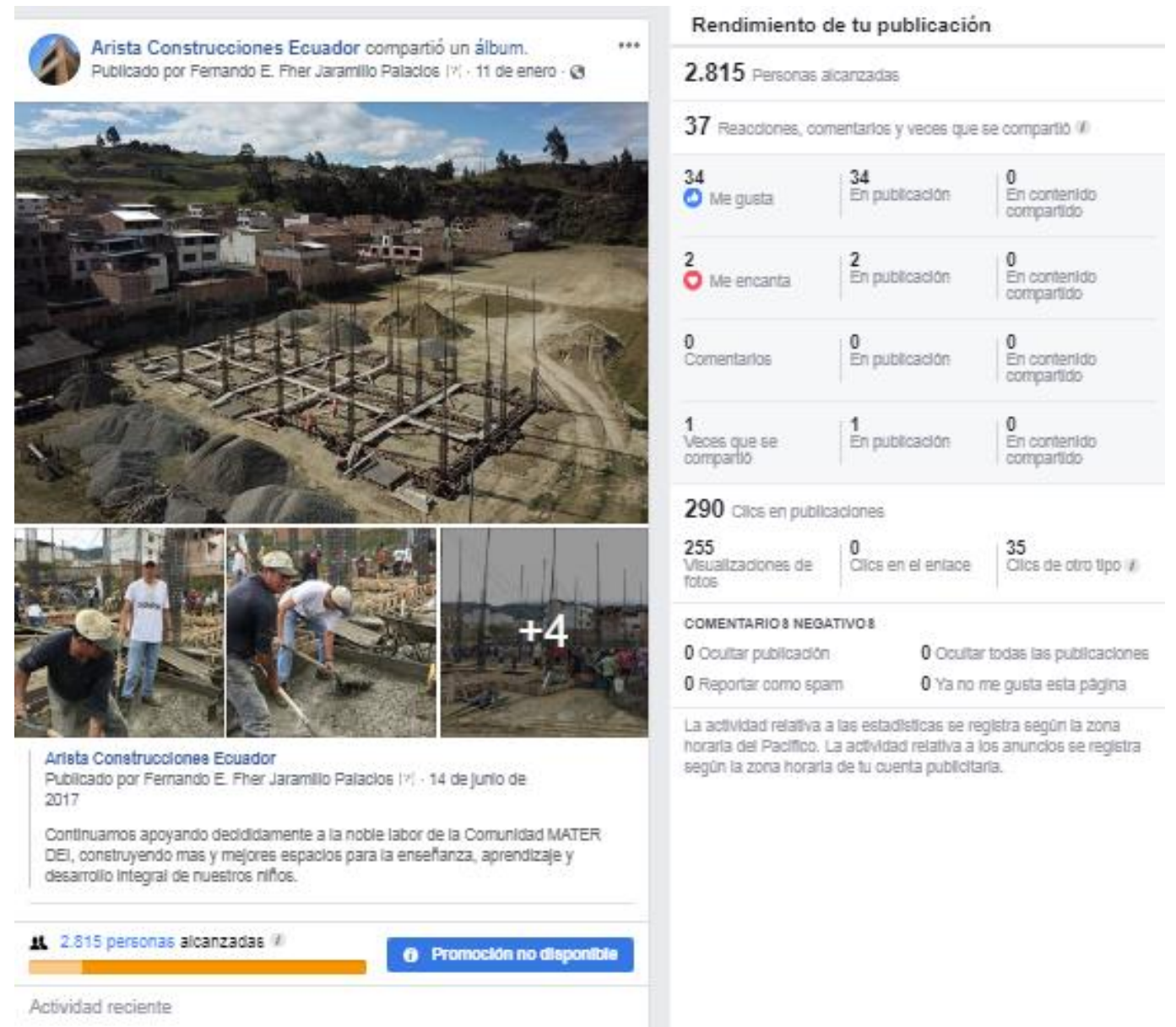

Fuente: Métricas obtenidas de la herramienta de Facebook Fan Page (2017)

\section{Discusión}

Los contenidos creados en las redes sociales y las herramientas de publicación de anuncios mejoran el alcance, más aún, cuando en combinación con el uso correcto de las palabras clave, el resultado denota mayor interacción. Lo que lleva a preguntarse y a pensar en un análisis futuro respecto de cómo potencializar el alcance de las publicaciones mediante el uso adecuado de dicha palabras.

\section{Conclusiones}

El alcance, impacto y utilidad de las redes sociales y herramientas de publicación de anuncios digitales para mejorar la promoción de servicios arquitectónicos en Loja está determinado por la constante actividad que genere el Gerente de la empresa dentro de las mismas.

La generación de contenido llamativo dentro de las publicaciones de las redes sociales incrementa la interacción de los usuarios con las publicaciones. 
El incremento de las interacciones de los usuarios con las publicaciones realizadas en las redes sociales, incrementa las ventas o el número de búsquedas de la empresa en Google Search.

La incidencia de usar la red social Google mi Negocio, permitió acceder a nuevos canales de promoción de manera directa, para que los clientes potenciales pueden buscar la empresa en su buscador y bajo un nivel de segmentación dado por ciudad.

A mayor número de anuncios publicitarios desarrollados, mayor número de personas que buscan y encuentran los servicios arquitectónicos pertenecientes a la empresa.

Las invitaciones orgánicas desde otros perfiles de Facebook a dar like a la fan page empresarial, no dan resultados favorables.

A mayor popularidad de la red social o la plataforma de publicación de anuncios publicitarios, mayor el impacto en el alcance del número de personas que la visualizan o interactúan con ella.

\section{Bibliografía}

ANUTO.NET. (2018, s.f. s.d.). ANUTO. Retrieved from https://anuto.net/las-10-mejores-paginasde-anuncios-gratis-en-espana

ANUTO.NET Ecuador. (2018, s.f. s.d.). ANUTO. Retrieved from https://anuto.net/las-10-mejorespaginas-de-anuncios-gratis-en-ecuador

Barker, M. S., Barker, D. I., Bormann, N. F., \& Neher, K. E. (2015). Marketing para medios sociales Un planteamiento estratégico. México D.F.: México Cengage Learning.

Cisneros Canlla, E. D. (2016). E-COMMERCE Comercio electrónico. Lima: Lima Macro EIRL.

Crespo, A. (2016). Marketing Digital. Madrid: España Marcombo.

Domínguez Doncel, A. (2010). Métricas del Marketing. Madrid: ESIC.

Gómez Vieites, Á., \& Otero Barros, C. (2013). Redes sociales en la empresa La revolución e impacto a nivel empresarial y profesional. Bogotá: Colombia Ediciones de la U.

Martí Parreño, J. (2011). Marketing y publicidad en internet básico. Madrid: España Starbook.

Multiplicalia. (2018). Multiplicalia. Retrieved from https://www.multiplicalia.com/redessociales-mas-usadas-2017/

OLX. (2018). Sobre OLX. Retrieved Julio 2, 2018, from OLX: https://help.olx.com.co/hc/es419/articles/212163106-Sobre-OLX

Rodríguez del Pino, D., \& Miranda Villalón, J. A. (2012). Publicidad on line Las claves del éxito en internet. Madrid: España ESIC. 
Ugarriza, D. (2013). Marketing y ventas por internet. Lima - Perú: Empresa Editora Macro.

Weber, L. (2010). Marketing en las redes sociales (2a. ed.).

Serrano, H. A. (2005). Todo internet: marketing digital y comercio electrónico. Retrieved from https://ebookcentral.proquest.com

Martínez, P. J. M., Martínez, S. J., \& Parra, M. M. C. (2015). Marketing digital: guía básica para digitalizar tu empresa. Retrieved from https://ebookcentral.proquest.com

Weber, L. (2010). Marketing en las redes sociales (2a. Ed.). Retrieved from https://www.mdconsult.internacional.edu.ec:2095

Kutchera, J. (2014). É-x-i-t-o: su estrategia de marketing digital en 5 pasos. Retrieved from https://ebookcentral.proquest.com 